\title{
Forecast for HEAT on Dome A, Antarctica: the High Elevation Antarctic Terahertz Telescope
}

Christopher K. Walker, Craig A. Kulesa, Dathon R. Golish, Abigail S. Hedden, K. Jacobs, et al.

Christopher K. Walker, Craig A. Kulesa, Dathon R. Golish, Abigail S. Hedden, K. Jacobs, Juergen Stutzki, J. R. Gao, Jacob W. Kooi, Dave Glaister, Willy Gully, Imran Mehdi, Mark R. Swain, Peter Siegel, "Forecast for HEAT on Dome A, Antarctica: the High Elevation Antarctic Terahertz Telescope," Proc. SPIE 5489, Ground-based Telescopes, (28 September 2004); doi: 10.1117/12.551424

Event: SPIE Astronomical Telescopes + Instrumentation, 2004, Glasgow, United Kingdom 


\title{
Forecast for HEAT on Dome A, Antarctica: The High Elevation Antarctic Terahertz Telescope
}

\author{
C. K. Walker ${ }^{a}$, C. A. Kulesa ${ }^{a}$, D. Golish ${ }^{a}$, A. Hedden ${ }^{a}$, K. Jacobs ${ }^{b}$, J. Stutzki $^{b}$, J. R. Gao $^{c}$, J. Kooi $^{d}$, \\ D. Glaister ${ }^{e}$, W. Gully ${ }^{e}$, I. Mehdi $^{f}$, M. Swain $f$, P. Siegel $f$ \\ ${ }^{a}$ University of Arizona, 933 N. Cherry Ave, Tucson, AZ, USA; \\ ${ }^{b}$ Universität zu Köln, Köln, Germany; \\ ${ }^{c}$ SRON, National Institute for Space Research, Groningen, The Netherlands; \\ ${ }^{d}$ California Institute of Technology, Pasadena, CA, USA; \\ ${ }^{e}$ Ball Aerospace, Boulder CO, USA; \\ $f$ Jet Propulsion Laboratory, Pasadena, CA, USA;
}

\begin{abstract}
We have proposed to develop a prototype 0.5 -meter far-infrared telescope and heterodyne receiver/spectrometer system for fully-automated remote operation at the summit of Dome A, the highest point on the Antarctic plateau. The unparalleled stability, exceptional dryness, low wind and extreme cold make Dome A a ground-based site without equal for astronomy at infrared and submillimeter wavelengths. HEAT, the High Elevation Antarctic Terahertz Telescope, will operate in the atmospheric windows between 150 and $400 \mu \mathrm{m}$, in which the most crucial astrophysical spectral diagnostics of the formation of galaxies, stars, planets, and life are found. At these wavelengths, HEAT will have high aperture efficiency and excellent atmospheric transmission most of the year. The proposed superheterodyne receiver system will be comprised of $0.8 \mathrm{THz}, 1.4 \mathrm{THz}$ and $1.9 \mathrm{THz}$ channels which will observe the pivotal $\mathrm{J}=7 \rightarrow 6$ line of $\mathrm{CO}$, the $\mathrm{J}=2 \rightarrow 1$ line of atomic carbon, and the far-infrared fine structure lines of $\mathrm{N}^{+}$and $\mathrm{C}^{+}$, the brightest emission lines in the entire Milky Way Galaxy. When combined with the HEAT telescope, the receiver system represents a uniquely powerful instrument for reconstructing the history of star formation in our Galaxy, with application to the distant Universe. The receiver system itself serves as a valuable testbed for heterodyne Terahertz components, using leading-edge mixer, local oscillator, low-noise amplifier, cryogenic, and digital signal processing technologies that will play essential roles in future Terahertz observatories. The proposed study will pave the way for future astronomical investigations from Dome A.
\end{abstract}

Keywords: Interstellar medium, heterodyne instrumentation, terahertz, star formation, molecular spectroscopy

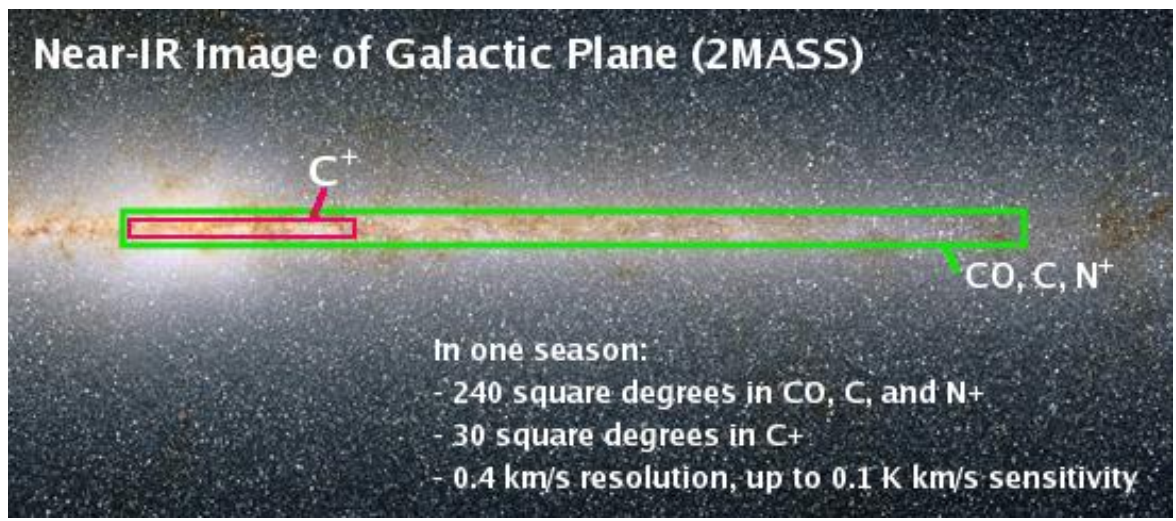

Figure 1. The power of HEAT: a definitive survey of the interstellar medium and star-forming clouds over 240 square degrees of the sky can be performed in the proposal period in the spectral lines of atomic carbon and $N^{\dagger}$. Over 30 square degrees of sky can be mapped in two months of the best winter weather in the pivotal $158 \mu \mathrm{m} \mathrm{C}^{+}$line. No other ground-based site is capable of routine observations at these highest frequencies.

Further information: Send correspondence to cwalker@as.arizona.edu or ckulesa@as.arizona.edu 


\section{SCIENTIFIC JUSTIFICATION}

Although great progress in understanding aspects of star and planet formation have resulted from the technological advent of sensitive (sub)millimeter wave detectors and the fabrication of large-format near-infrared detector arrays, many fundamental aspects of the formation and evolution of galaxies, stars, planets, and life remain shrouded in uncertainty. The common element of these mysteries is the evolution of interstellar clouds of dust and gas, from which stars and planets are born, and to which stars return enriched material at the end of their lives. These clouds sculpt the evolution of entire galaxies. The far-infrared (FIR) contains the brightest and most diagnostic spectral lines of the entire electromagnetic spectrum; in particular the pivotal fine structure lines of $\mathrm{C} \mathrm{N}^{+}$and $\mathrm{C}^{+}$at $0.8,1.4$ and $1.9 \mathrm{THz}(370,205$ and $158 \mu \mathrm{m})$, respectively. Only on the high Antarctic plateau is the atmosphere dry, cold and stable enough to permit survey observations at all three wavelengths. In performing a Galactic Plane survey of these spectral lines, fundamental new insights into Galactic evolution, and star formation will be pioneered (Figure 1). In particular, HEAT will:

1. Directly witness the formation of interstellar molecular clouds for the first time, and answer where and how cloud formation takes place in the context of the Galaxy as a whole, with direct impact on star formation and Galaxy evolution. Natal molecular clouds are mostly comprised of spectroscopically elusive $\mathrm{H}_{2}$, but the principal forms of carbon in forming will be $\mathrm{C}^{+}$and $\mathrm{C}$ - not $\mathrm{CO}$, as found in developed clouds. 2MASS J-H/H-K extinction maps, in comparison with existing $\mathrm{CO}$ surveys, will guide the selection of fields for mapping.

2. Derive a definitive star formation rate as a function of radius in the plane of the Milky Way, providing an optimum set of data to calculate the Schmidt Law in the Galaxy. $\mathrm{N}^{+}$emission at $1.4 \mathrm{THz}$ provides a measure of the ionizing luminosity with unmatched sensitivity, angular and spectral resolution, and is unaffected by extinction.

3. Provide the first map of warm dense molecular gas via $\mathrm{CO}$ emission in the $\mathrm{CO} \mathrm{J}=7 \rightarrow 6$ line at $0.8 \mathrm{THz}$, which can be observed simultaneously with the $\mathrm{J}=2 \rightarrow 1$ fine structure line of neutral carbon. Measurement of this energetic gas probes the pivotal feedback of stars with their parent interstellar clouds. It is energetic molecular gas that participates in molecular outflows, is the best probe of radiation fields at the surfaces of clouds, and is warmed by star formation in cloud cores.

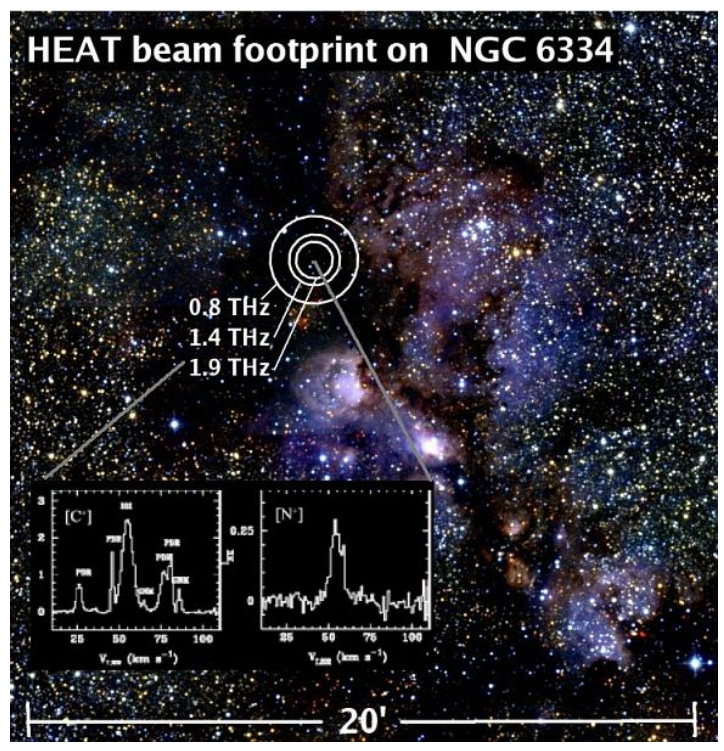

Figure 2. The 3 beams of HEAT overlaid upon a $2 M A S S$ image of NGC 6334. The size of the cospatial 0.8, 1.4, and $1.9 \mathrm{THz}$ beams is $3,1.7$, and 1.3 arcminutes, respectively.

The adoption of heterodyne receivers for HEAT naturally provides the high spectral resolution $\left(<0.4 \mathrm{~km} \mathrm{~s}^{-1}\right)$ needed to disentangle kinematically the many cloud components along any particular line of sight through the Galactic Plane. The angular resolution provided by the 0.5-meter clear aperture (Figure 2) is optimized for resolving individual cloud components throughout the Galaxy while providing adequate mapping speed to perform a substantial survey of the Galaxy in a single observing season. The entire Galactic Plane visible from Dome A $\left(+10^{\circ}<l<-120^{\circ}\right)$ will be mapped in 6 
months to $1.5^{\prime}$ resolution and RMS sensitivity of $\sim 0.1 \mathrm{~K}$ at $\mathrm{CO}$ and $\mathrm{C}$, and $<1 \mathrm{~K}$ at $\mathrm{N}^{+}$, assuming that observations are being performed $75 \%$ of the time. A total of $\sim 30$ square degrees can be mapped in $\mathrm{C}^{+}$during the coldest two months (typically August-September) to a resolution of $0.7^{\prime}$ and a sensitivity of $\sim 1$ K RMS (Figure 1).

\section{INSTRUMENT DESCRIPTION}

HEAT will be a fully automated, state-of-the-art THz observatory designed to operate autonomously from Dome A in Antarctica. The combination of high altitude $(4,200 \mathrm{~m})$, low precipitation, and extreme cold make the atmospheric transmission in the FIR exceptionally good from this site. In Figure 3 we present a plot of the expected atmospheric transmission above Dome A as a function of wavelength (Lawrence 2004), indicating that winter weather at Dome A approaches (within an order of magnitude) the quality of that achieved by SOFIA. The wavelengths of several important astrophysical lines are indicated with arrows. HEAT is designed to take advantage of these unique atmospheric conditions and observe simultaneously in [C II] $(158 \mu \mathrm{m})$, [N II] $(205 \mu \mathrm{m})$, and CO J $=7 \rightarrow 6 /[\mathrm{C} \mathrm{I}] 2 \rightarrow 1(370 \mu \mathrm{m})$.

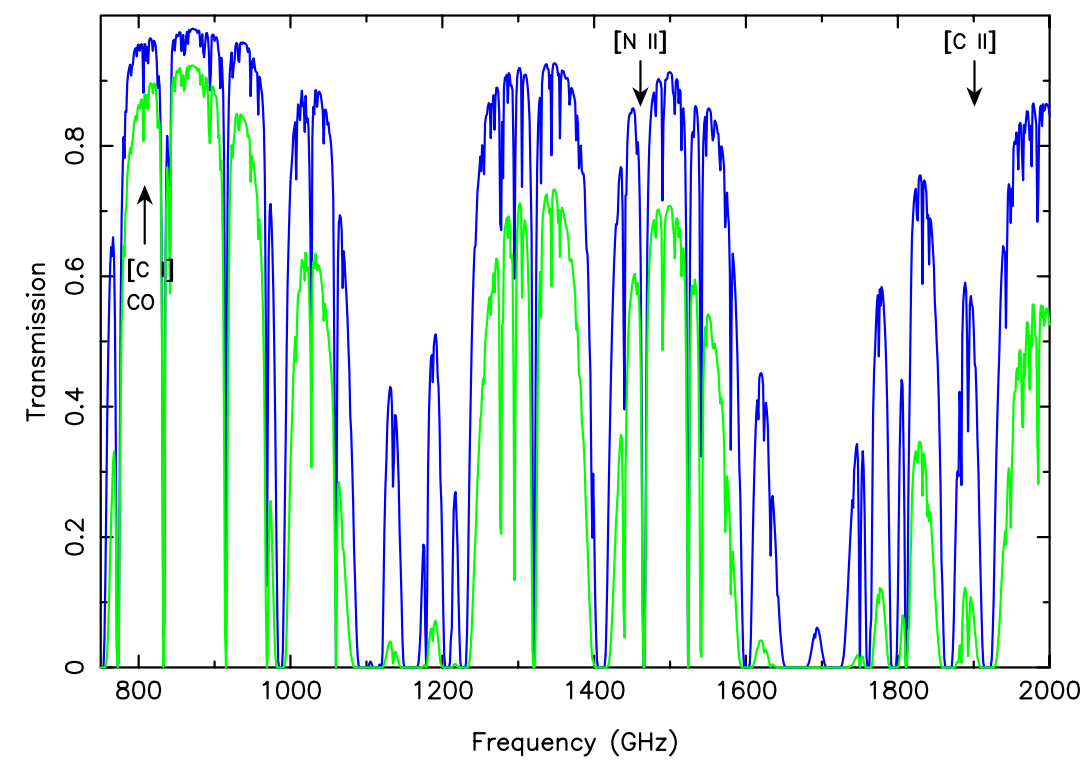

Figure 3. Atmospheric transmission in the far infrared/submillimeter, derived from the PWV estimates for Dome A of Lawrence (2004). The pwv content for each model atmosphere is 120 and 30 microns respectively, corresponding to $\sim 75$ percentile and $\sim 10$ percentile winter weather at Dome A. Arrows indicate the wavelengths of the [N II], [C II], and CO/[C I] lines.

A conceptual drawing of HEAT is shown in Figure 4. For robustness and efficiency, the telescope and instrument are integrated into a common optical support structure (OSS). HEAT will be mounted on top of a University of New South Wales AASTINO (Automated Astrophysical Site-Testing InterNational Observatory). The AASTINO provides power and communications for the HEAT telescope and instrument. The HEAT/AASTINO facility is functionally equivalent to a space-based observatory. Indeed, many of the key components used in the instrument were originally developed for space applications.

\subsection{Telescope}

The telescope is designed to have maximum efficiency and the minimum number of optical components. Its design is similar to that of Kraus (1966). Incoming light is reflected horizontally off a $45^{\circ}, 0.5 \times 0.7 \mathrm{~m}$ flat reflector to an f/5 off-axis parabolic mirror. The converging beam is intercepted by a flat tertiary mirror that directs it into the receiver. The tertiary mirror can chop the incoming beam between source and reference positions $\left(\Delta \mathrm{az} \sim 10^{\prime}\right)$ at a rate of 0 to $4 \mathrm{~Hz}$. All three mirrors are fabricated from aluminum on a numerical milling machine and have a surface roughness $\leq 3 \mu \mathrm{m}$ rms. Elevation tracking is achieved by rotating the first flat reflector. Azimuth tracking is achieved by rotating the OSS on a bearing attached to the roof of the AASTINO. The absolute pointing accuracy will be 15 ", $1 / 5$ of the smallest diffractionlimited beam. The slew speed will be $1^{\circ}$ per sec. Vertex-RSI has reviewed the proposed design and provided a cost estimate for the delivery of a telescope meeting these specifications. The cost estimate, which includes detailed design, fabrication, and testing of the telescope and drive system, is provided in the budget.

To prevent ice accumulation, the telescope is enclosed and warmed by waste heat forced up through the azimuth bearing from the AASTINO. A small radome made of a low-loss dielectric (e.g. Goretex or polyethylene) encircles the first flat 


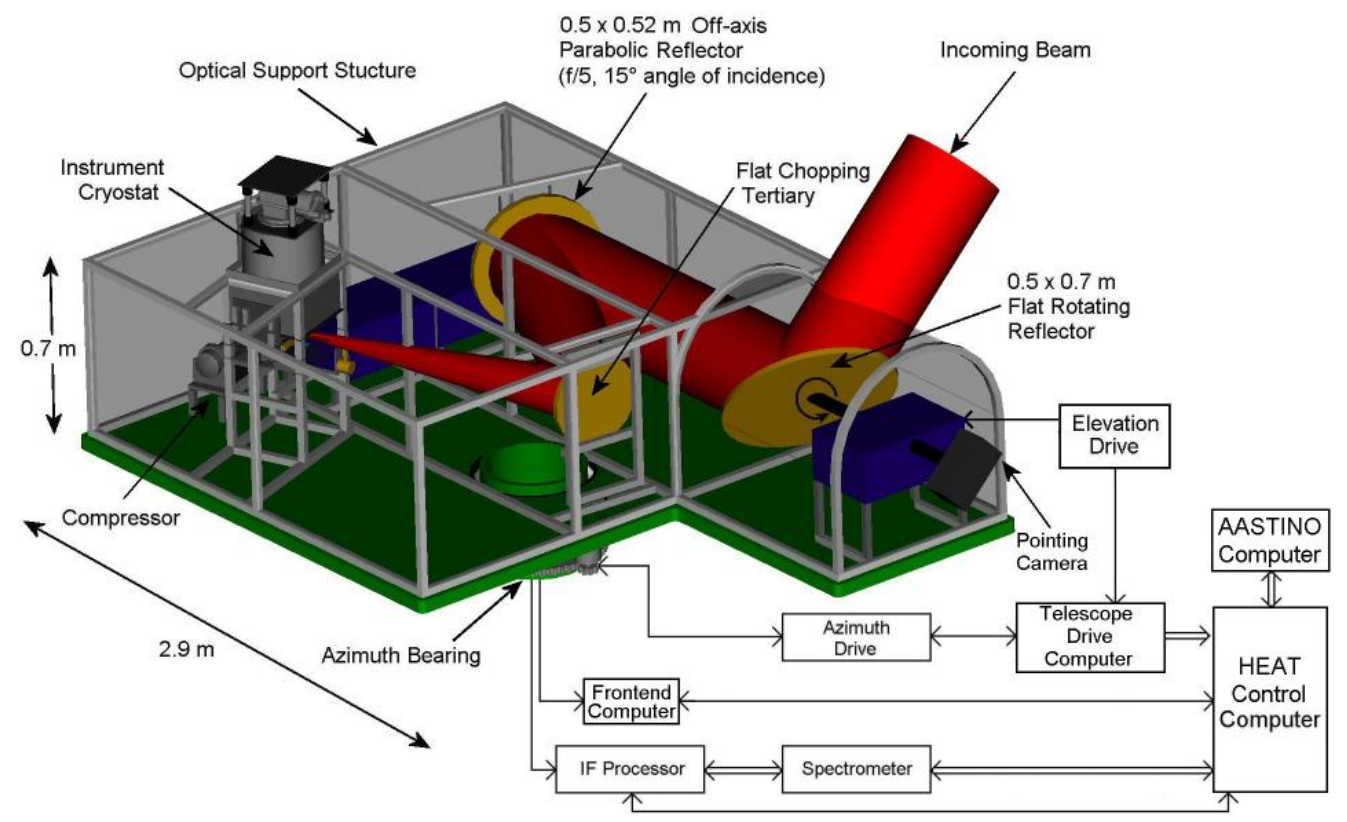

Figure 4. HEAT concept: The telescope has an effective collecting area of $0.5 \mathrm{~m}$. Elevation tracking is accomplished by rotating the $45^{\circ}$ flat reflector. The entire telescope structure is warmed by waste heat from the AASTINO module below and rotates for azimuth tracking. The HEB and SIS mixers used in the instrument package are cooled to $\sim 4 \mathrm{~K}$ using closed-cycled cryo-cooler technology developed for space-based applications.

reflector. This optical configuration provides an unobstructed view of the sky. A CCD camera (provided by UNSW) and 1-5.3 $\mu \mathrm{m}$ IR camera are mounted just outside the radome on an extension of the elevation axis. The cameras provide optical pointing and crucial site testing data.

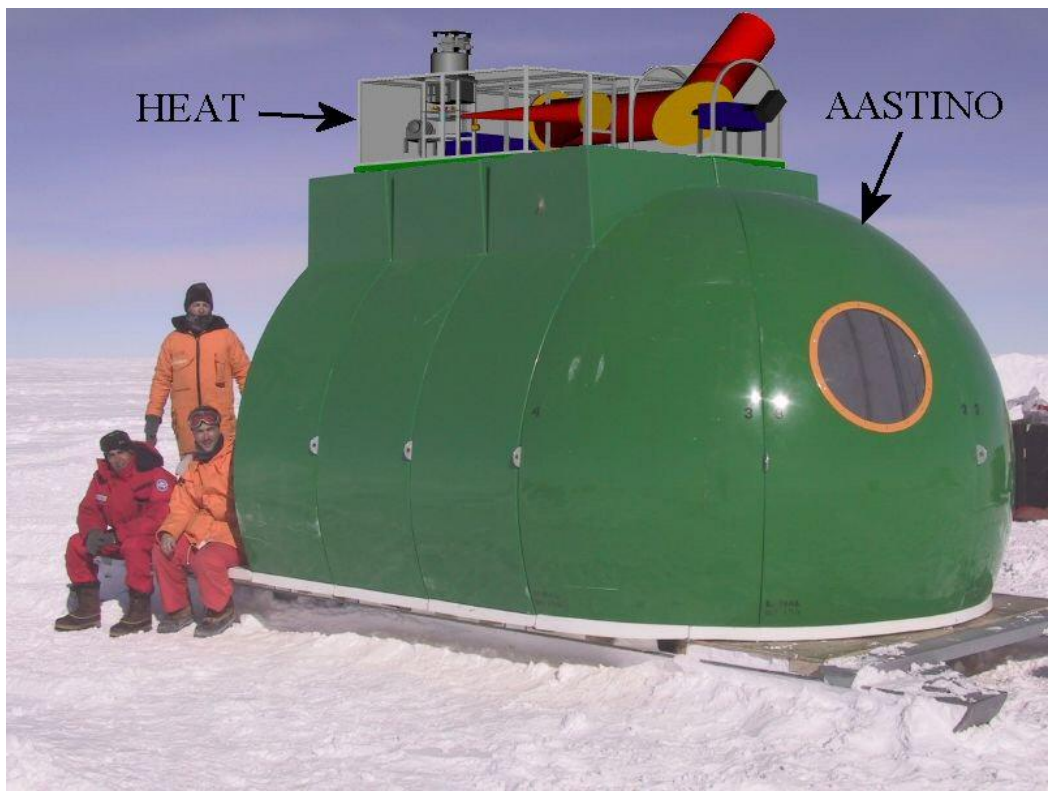

Figure 5. Rendering of HEAT atop a UNSW AASTINO 


\subsection{Receiver}

\subsubsection{Design Approach}

Heterodyne receivers are needed to achieve the sensitive, high spectral resolution $\left(R=\lambda / \Delta \lambda>10^{6}\right)$ observations of $[\mathrm{N} \mathrm{II}],[\mathrm{C} \mathrm{II}]$, and $\mathrm{CO} /[\mathrm{C} \mathrm{I}]$ required for the proposed Galactic plane survey. The key components of a submillimeter-wave heterodyne receiver are the mixer and local oscillator (LO). There are 3 types of mixers in common use; the Schottky diode mixer, the SIS mixer, and, more recently, the Hot Electron Bolometer (HEB) mixer. The Schottky diode mixer is less sensitive than either SIS or HEB mixers and requires orders of magnitude more LO power. Their principal advantages are robustness and ability to operate at room temperature. Below $\sim 1 \mathrm{THz}$ SIS mixers provide the lowest receiver noise temperatures $\left(T_{r e c} \leq 400 \mathrm{~K}\right)$. Above $1 \mathrm{THz}$ HEB mixers have the best performance $\left(T_{r e c} \leq 900 \mathrm{~K}\right)$. The $\sim 1 \mathrm{THz}$ upper limit to SIS mixer performance is currently set by the availability of suitable superconducting materials. Therefore, HEAT will use an SIS mixer for the CO/[C I] channel and HEB mixers for the [N II] and [C II] channels. The specific mixers and LO's that will be used in HEAT were originally developed for use in the HIFI instrument for the Hershel Space Observatory.

\subsubsection{Receiver Optics}

A close-up of the receiver optics is shown in Figure 6. The incoming beam from the folding tertiary encounters two bandpass filters, the first centered on the [N II] $(1.459 \mathrm{THz})$ line and the second on the [C II] $(1.9 \mathrm{THz})$ line. Outside of their nominal passband, the filters are highly reflective. Therefore, when the incoming beam encounters the first filter, all but a narrow range of frequencies around the [C II] line are reflected to the [N II] filter. The light reflected off the [N II] filter contains the $\mathrm{CO} \mathrm{J}=7-6$ and $\left[{ }^{12} \mathrm{C} \mathrm{I}\right]$ and $\left[{ }^{13} \mathrm{C} \mathrm{I}\right] \mathrm{J}=2-1$ lines. The three emerging beams are collimated and directed into the instrument cryostat. Before entering the cryostat, the corresponding LO signals are injected into the collimated beams using Mylar (for the $810 \mathrm{GHz}$ mixer) and silicon (for the 1.45 and $1.9 \mathrm{THz}$ mixers) beam splitters. The Mylar is used to inject the $810 \mathrm{GHz}$ LO because it is both low-loss and wideband, permiting DSB operation at the low IF frequency $(1.5 \mathrm{GHz})$ required for simultaneous observations of $\mathrm{CO}$ and $[\mathrm{CI}]$ lines. Silicon etalons can serve as efficient LO diplexers, passing $>95 \%$ of the signal while reflecting $>50 \%$ of the LO power (Mueller and Waldman 1994). These properties make silicon etalons attractive beam splitters for THz mixers where LO power is precious and the size and complexity of MartinPuplett diplexers can be prohibitive. The IF frequency of the [C II] and [N II] mixers is $3 \mathrm{GHz}$. Upon entering the cryostat, the beams pass through infrared blocking filters. For the HEB mixers, the same type of bandpass filters used for frequency diplexing will be used at the entrance window of the $4 \mathrm{~K}$ radiation stage. Here the filters not only prevent infrared loading on the $4 \mathrm{~K}$ stage, they also help to prevent the HEB mixers from being saturated by the incoming radiation field.

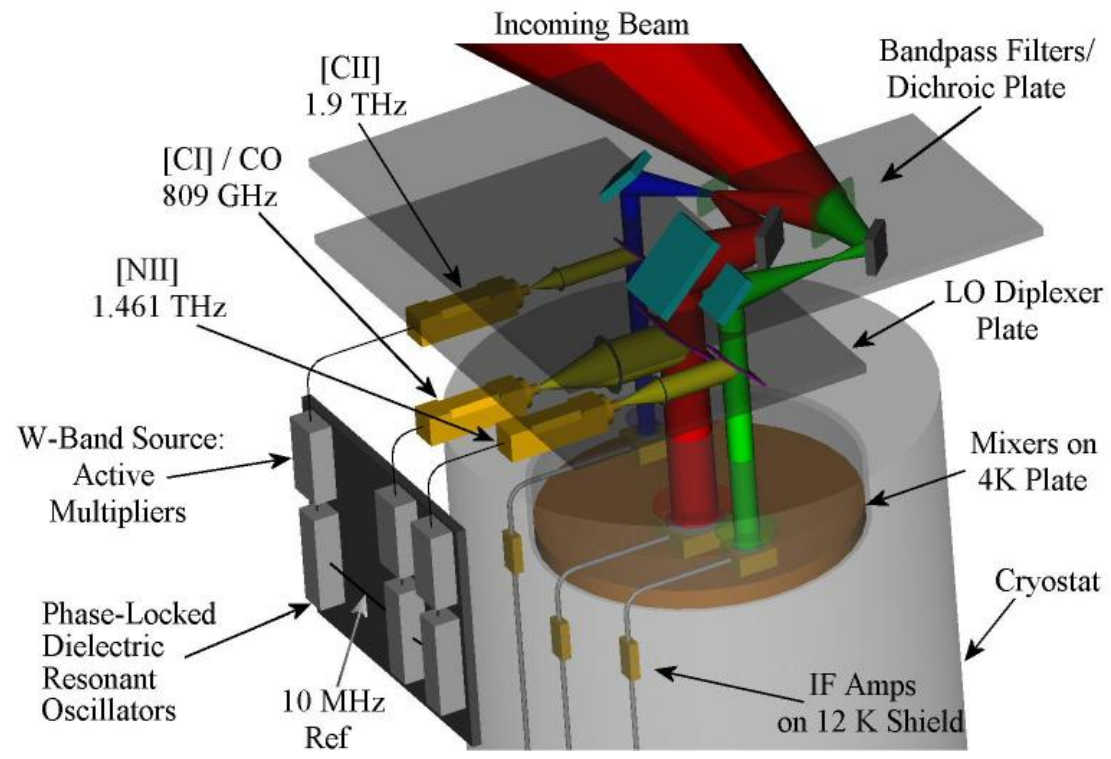

Figure 6. Optical subsystem and LO injection for HEAT's HEB and SIS mixers. 


\subsubsection{Mixer Performance}

The HEB mixers for HEAT will be provided by SRON/Delft and follow the successful quasi-optically coupled, NbN phonon-cooled design of Gao et. al. (2004). These mixers have the lowest noise temperatures and LO power requirements of any mixers measured to date $\left(T_{r e c}=940 \mathrm{~K}\right.$ and $P_{L O}=0.17 \mu \mathrm{W}$ at $\left.1.89 \mathrm{THz}\right)$. A plot summarizing the noise performance of HEB mixers world-wide is shown in Figure 7. Recent Allan variance measurements (Kooi 2004) of HEB stability (Figure 8) show excellent performance can be achieved if the incoming beam is chopped at $\geq 1 \mathrm{~Hz}$. The HEAT telescope is designed to accommodate sky chopping at frequencies up to $\sim 4 \mathrm{~Hz}$.

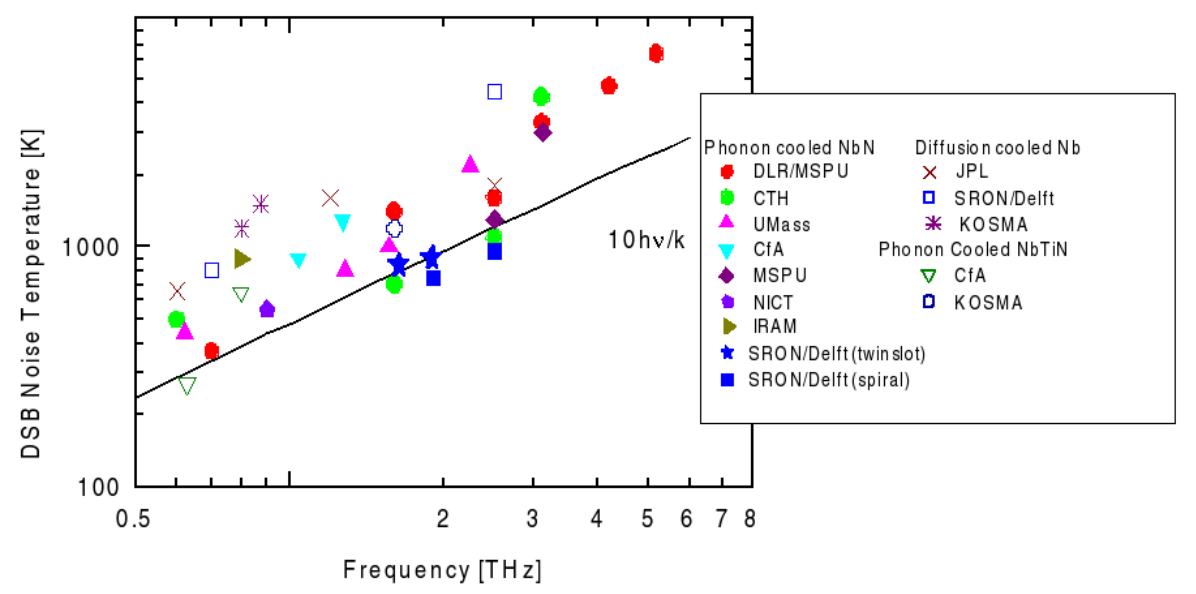

Figure 7. Comparison of measured HEB mixer noise temperatures worldwide (from Gao et al. 2004)

The SIS mixer will be provided by the University of Cologne. The design and fabrication of the SIS mixer is described by Puetz et al. (2004, this volume) and has been successfully used on the AST/RO telescope at the South Pole for several years. The noise temperature is expected to be $\sim 200 \mathrm{~K}$.

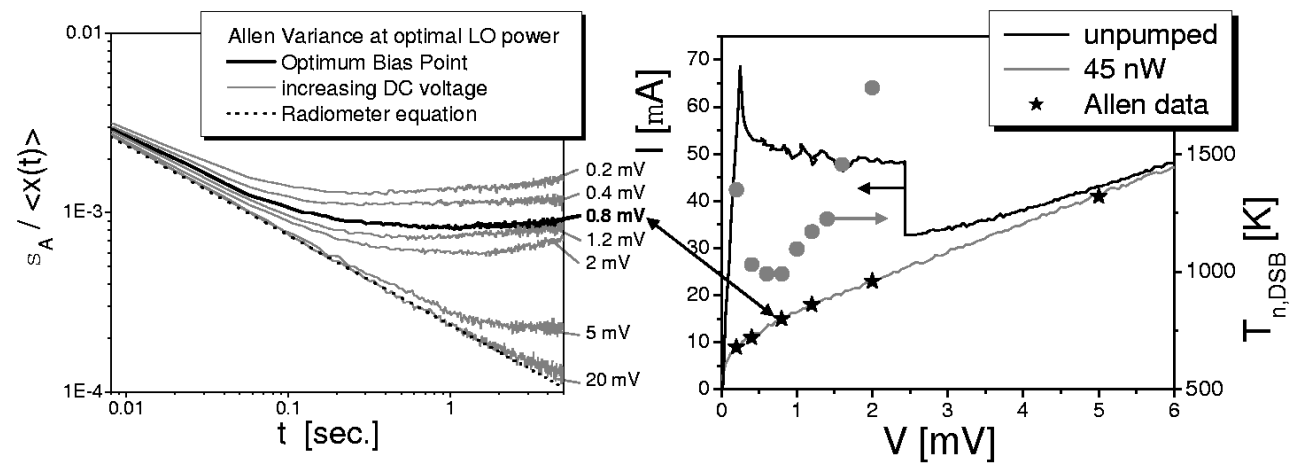

Figure 8. Allan variance stability tests for Delft HEB mixers are in accordance with the radiometer equation if a chopping secondary is used.

\subsubsection{Local Oscillators}

HEAT will employ 3 solid-state local oscillator chains built by the Jet Propulsion Laboratory. The $809 \mathrm{GHz}$ chain will consist of three doublers. The $1.45 \mathrm{Thz}$ chain will have four doublers, and the $1.9 \mathrm{THz}$ chain will be comprised of one doubler and two triplers. These chains leverage heavily from the technology developed for Herschel and have had their performance validated in lab measurements. All three chains easily meet our LO power requirements of $>100 \mu \mathrm{W}$ at $809 \mathrm{GHz}$ and $>1 \mu \mathrm{W}$ at 1.46 and $1.9 \mathrm{THz}$. The JPL LO chains will be pumped by CTI/Millitech DRO (dielectric resonant oscillator)/active multiplier chains that can source $\sim 3 \mathrm{dBm}$ of power between 90-106 GHz. Each of the phase-locked 
DRO's is fixed tuned to a single frequency, that when up-converted, corresponds to one of the three target astrophysical lines. A CAD drawing illustrating the integration of the LO's is provided in Figure 6. Since each of the three receivers acts independently, any increase in phase-noise that might occur by using an active multiplier chain has minimal, if any, impact on the quality of the collected data. The frequency stability of the LO chains will be better than $\pm 0.5 \mathrm{MHz}$ at $809 \mathrm{GHz}$ and $\pm 1.0 \mathrm{MHz}$ at 1.46 and $1.9 \mathrm{THz}$.

\subsubsection{IF Processing \& Spectrometers}

The IF output of the HEB mixers is centered at $5 \mathrm{GHz}$. In order to simultaneously detect the CO $J=7 \rightarrow 6$ line in the lower sideband and the [C I] line in the upper sideband, the SIS mixer will have an IF center frequency of $1.5 \mathrm{GHz}$. The IF output of each mixer is amplified by a low-noise MMIC amplifier and downconverted to baseband for processing by three, 1024 lag autocorrelators. The autocorrelators for the [N II] and [C II] channels will each have $2 \mathrm{GHz}$ of instantaneous bandwidth and $\sim 2 \mathrm{MHz}$ resolution. The autocorrelator for the lower frequency $\mathrm{CO} /[\mathrm{C} \mathrm{I}]$ channel will run at half the clockspeed of the other two channels, yielding an instantaneous bandwidth of $1 \mathrm{GHz}$ and frequency resolution of $\sim 1 \mathrm{MHz}$ resolution. These correlators provide velocity coverage in access of $316 \mathrm{~km} / \mathrm{s}$ at a resolution $\leq 0.4 \mathrm{~km} / \mathrm{s}$, enabling all three lines to be resolved and observed throughout the Milky Way without needing to retune. Spaceborne Inc. of La Canada, CA has demonstrated a $2 \mathrm{GHz}$ wide, 1024 lag correlator that meets our design specifications (see Figure 9) and provided a quote for the complete HEAT correlator system.
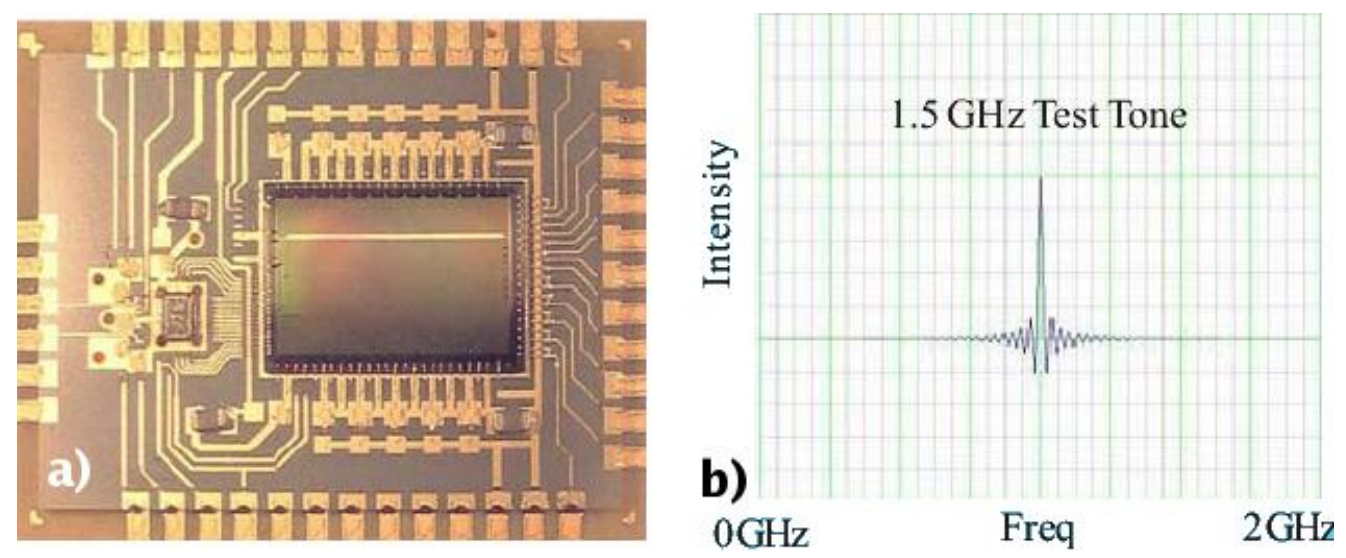

Figure 9. The Spaceborne digital autocorrelator provides an ideal "off-the-shelf” multichannel spectrometer for HEAT. a) 2 GHz Spaceborne correlator chip. b) Laboratory measurement of spectral response.

\subsubsection{Cryogenics}

A reliable, efficient, low-vibration $4 \mathrm{~K}$ cryogenic system is essential to the success of HEAT. Experience has shown that care must be taken to insure the uninterrupted operation of cryogenic systems over extended periods. This philosophy is even more true for the proposed remote, automated facility at DOME A. Indeed, the system requirements for HEAT are more like those of a spaceraft than a ground-based observatory. It is for this reason that we have a teamed with Ball Aeropace to adapt one of their successful spacebased cryogenic systems for work on HEAT.

\section{HEAT Cryogenic Subsystem Trades and Approach}

The cryogenic subsystem for HEAT must maintain the detectors at $4 \mathrm{~K}$. A number of approaches, including off-theshelf commercial cryocoolers (or mechanical refrigerators), were evaluated against the system requirements. The need for unattended operation for at least 1 year eliminates the possibility of using liquid expendable cryogens. The remote and harsh Antarctic environment prevents the use of off-the-shelf commercial cryocoolers primarily due to limited power for HEAT $(<600 \mathrm{~W}$ total $)$ provided by the AASTINO. High cost prevents the use of fully space qualified cryocoolers for HEAT. The resulting optimum cryogenic system that meets both the technical and cost requirements is a combination of commercial and aerospace cryocooler hardware. The HEAT cryogenic system requirements are shown in Table 1.

After lifetime, the most demanding requirement is the input power. To meet the power requirement, we have combined improvements in a number of areas: 
Table 1. Cryogenic system requirements for HEAT

\begin{tabular}{cc}
\hline \hline Requirement & Specification \\
\hline 4K cooling capacity & $4 \mathrm{~mW}$ \\
14 K cooling capacity & $220 \mathrm{~mW}$ \\
70 K cooling capacity & $3.6 \mathrm{~W}$ \\
4 K temperature stability & $\pm 0.01 \mathrm{~K}$ \\
Input power & $<500 \mathrm{~W}$ \\
Lifetime & 2 years total with 1 year maintenance interval \\
Vibration & Low \\
Vacuum level & $<1 \times 10^{-5}$ torr at 1 year \\
Cost & Low to Moderate \\
\hline
\end{tabular}

Table 2. Cryocooler approaches and applicability to HEAT

\begin{tabular}{cccc}
\hline \hline Capacity at 4K & Manufacturer & Input power (Watts) & Meets Requirement? \\
\hline $0.1 \mathrm{~W}(\mathrm{G}-\mathrm{M})$ & Sumitomo SRDK-101D & 1300 & No \\
$0.5 \mathrm{~W}(\mathrm{G}-\mathrm{M})$ & Sumitomo SRDK-205D & 3500 & No \\
$0.5 \mathrm{~W}$ (Pulse Tube G-M) & Sumitomo SRP-052A & 7500 & No \\
$20 \mathrm{~mW}($ Hybrid G-M/J-T) & Ball Aero - HEAT & 400 & Yes \\
\hline
\end{tabular}

1. The cooler is part of a custom cryostat that is designed to take advantage of the low environmental temperatures to minimize heat load parasitics and save power.

2. The vacuum-sustaining pumping system ( $~ 150 \mathrm{~W}$ input) has been eliminated by using the cryocooler as a cryopump.

3. To circumvent the problem that makes traditional mechanical coolers at $4 \mathrm{~K}$ so power inefficient, our design uses two cooling cycles, each to best advantage. The G-M cools down to $14 \mathrm{~K}$, where its regenerator is still efficient. Then, a J-T cold head is used for the remaining step down to $4 \mathrm{~K}$. To minimize complexity, both systems run off the same compressor. The G-M is a modified commercial model. The J-T system only consists of a few passive components and is highly leveraged off our space cooler hardware (demonstrated $3.4 \mathrm{~K}$ cooling).

4. The off-the-shelf G-M cooler is modified to take advantage of the low ambient temperatures. The standard CTI Model 350 cold head is used, but the compressor is replaced with a commercial oil-free linear compressor, which will operate in the colder environment and provide additional temperature control.

Table 2 shows a compilation of cryocooler approaches and their power consumption. Only the hybrid system can consume less than the $500 \mathrm{~W}$ requirement. The $4 \mathrm{~K}$ refrigeration requirement for the commercial 2-stage coolers must be $>0.25 \mathrm{~W}$ because they don't have a $14 \mathrm{~K}$ stage available to help reduce parasitic loads. The Pulse Tube G-M has a number of attractive features (e.g. low maintenance and vibration), however low efficient makes its power consumption greater than a standard G-M cooler and, therefore, not suitable for HEAT. The extra cost of the semi-custom cryosystem enables the great reduction in input power needed for operation from a remote site.

The HEAT system requires low cryocooler induced vibration levels to minimize microphonic effects. The HEAT J-T cold head inherently results in very low cryocooler induced vibration as it employs long flexible tubes coming from the compressor. The tubing allows for the isolation of the compressor vibration from the detectors, leaving only the completely passive J-T cold head components (essentially orifices and tubing) in contact with the detectors. Commercial G-M cold heads produce significant vibration and even Pulse Tube versions can cause issues due to the oil-canning (from the pressure 
waves) of the cold-finger. Thus, the HEAT approach will produce significantly lower vibration levels than the commercial alternatives.

\section{Cryo System Implementation}

The proposed cooler has a high degree of technology maturity. All of the cryocooler components and their integration have been proven in test. The foundation of the cryocooler design is component and system development proven in test verification from Ball programs such as the Active Cooler Test and Development Program (ACTDP) and JPL Planck programs. The Ball ACTDP Study Phase program fabricated, integrated, and tested the cryocooler components. Cooling capacities in excess of $25 \mathrm{~mW}$ were measured for temperatures down to $5 \mathrm{~K}$. Tests with ${ }^{3} \mathrm{He}$ as the J-T working fluid extended the temperatures down to $3.5 \mathrm{~K}$, and demonstrated $12 \mathrm{~mW}$ of cooling at $3.8 \mathrm{~K}$, more than adequate for cooling HEAT's complement of HEB mixers.

Leveraging off Ball Aerospace's system and instrument expertise, the cryogenic system has been designed to meet the HEAT's unique requirements. A block diagram of the HEAT cryogenic system is shown in Figure 10.

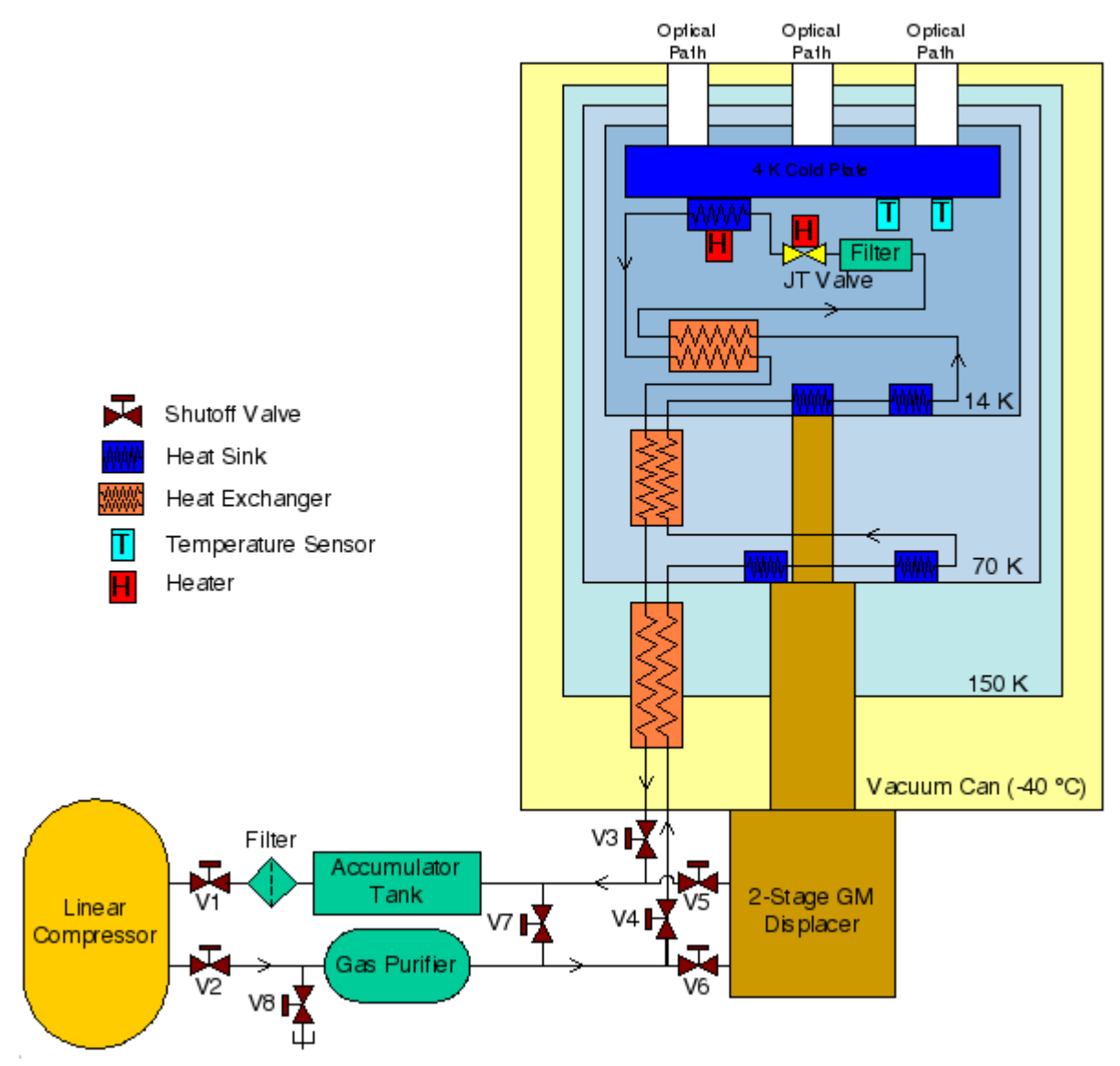

Figure 10. The cryocooler supporting the HEAT cryogenic system is a hybrid cooler consisting of a modified Gifford McMahon precooler and a Joule-Thomson cooler.

The $4 \mathrm{~K}$ temperature control as well as the cryocooler on/off and a safety defrost heater will be handled by a commercial temperature controller from Lake Shore Cryotronics. A trim heater, providing just a few milliwatts of power, will maintain the $4 \mathrm{~K}$ temperature with changing environmental conditions. The analog output will provide remote operation of a safety defrost heater used in case the JT orifice plugs. The relay will allow the cryocooler to be remotely turned on or off.

To comply with the tight vibration and thermal requirements, Ball will provide a separate structural mount for the detector package, and use the J-T to refrigerate the isolated system (see Figure 11). The temperature of the cold plate will be actively regulated to within $10 \mathrm{mK}$. To meet the tight power constraints, Ball has designed a special dewar that 
uses the cold Antarctic environment to minimize power consumption. This type of optimization and design strategy is common for space missions where power conservation and reliability are essential. To our knowledge this is the first time this philosophy is being employed to such a degree on a ground-based instrument.

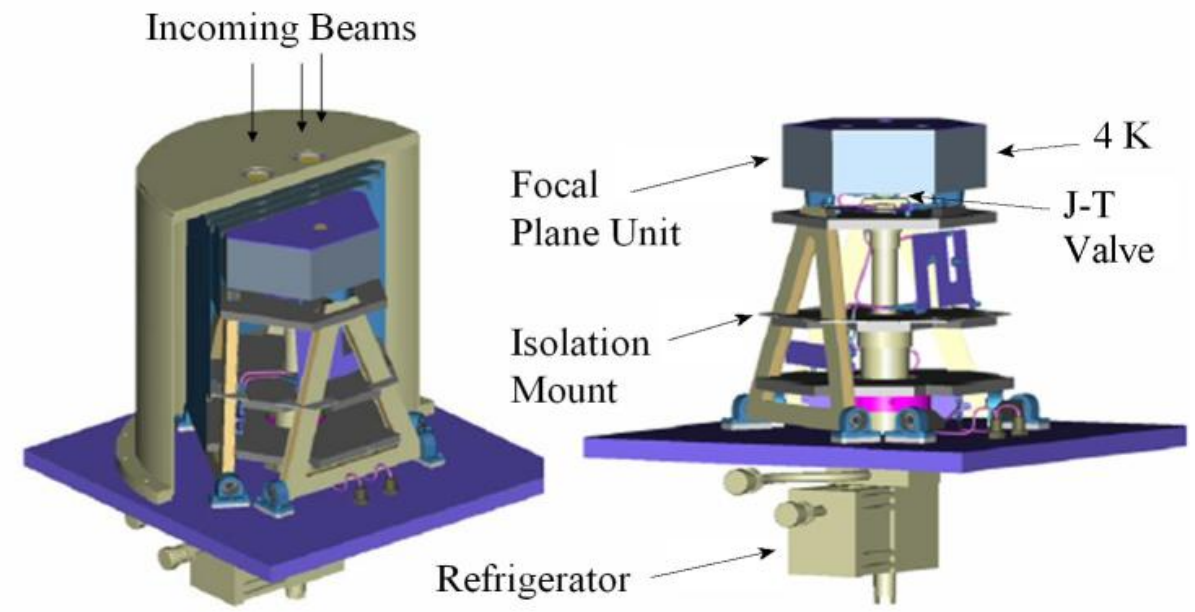

Figure 11. Cut-away views of low-vibration, long-life, $4 \mathrm{~K}$ closed-cycle cryostat designed by Ball.

\subsubsection{Calibration}

HEAT will be able to calibrate observations through several means. 1) A vane with an ambient temperature absorbing load will be located at the dewar entrance window, allowing standard chopper wheel calibration to be performed. 2) HEAT will routinely perform sky-dips to compute the atmospheric optical depth in each of it's three wavelength bands. 3) HEAT will regularly observe a standard list of calibration sources. 4) The AASTINO will also host a Fourier Transform Spectrometer (funded through the Australian program) that will measure atmospheric transmission throughout the FIR. These measurements will be coordinated with HEAT spectral line observations to provide cross calibration.

\subsubsection{Control System and Communication}

The HEAT control system will consist of a centralized control computer that communicates with the telescope drive system, receiver frontend (e.g. mixers, LO's, and cryostat) computer, backend (spectrometer) computer, and the AASTINO via Ethernet. HEAT will be designed to work autonomously for up to a week at a time, performing pre-programmed observational programs and storing astronomical and housekeeping data in non-volatile memory. Preprocessed sample data will be uploaded to control centers at the Universities of Arizona and New South Wales via (1-3) dedicated Iridium satellite channels. Raw data will be recovered from HEAT each year during maintenance and processed into the released data products.

\section{CONCLUSION}

With the implementation of HEAT, the astronomical community will have a new, powerful capability for exploring the origin of stars, galaxies, and planetary systems like our own. It will serve as a model for future Antarctic observatories and the first step toward realizing the research potential of Dome A. We look forward both to the exciting science that will come from HEAT and the challenge of making it a reality. 


\section{References}

[1] Gao, J. R., Hajenius, M., Baselmans, J., Klawijk, P., de Korte, Voronov, B., and Gol'tsman, G., 2004, "NbN Hot Electron Bolometer Mixers with Superior Performance for Space Applications", International Workshop on Low Temperature Electronics, 23-24 June 2004, (invited paper).

[2] Kooi, J., 2004, private communication.

[3] Krauss, J., "Radio Astronomy”, 1966, McGraw-Hill, NY

[4] Lawrence, J. S., 2004, "Infrared and submillimetre atmospheric characteristics of high Antarctic plateau sites", PASP, in press. 